\title{
ANÁLISE DE VAZÕES DA BACIA HIDROGRÁFICA DO RIO PARAÍBA DO SUL, VARIABILIDADES, INTENSIDADES E PERÍODOS DE RETORNO
}

\author{
Eduardo Soares LeiteLuiz ${ }^{1}$, Antônio de Oliveira ${ }^{2}$ \\ 1'Instituto de Geografia/Universidade Federal de Uberlândia/ eduardo.geoufu@ hotmail.com \\ ${ }^{2}$ Instituto de Geografia/Universidade Federal de Uberlândia/ \\ luiz.oliveira@ufu.br
}

Eixo: 3 - Climatologia em diferentes níveis escalares: mudanças e variabilidades

\begin{abstract}
Resumo
A utilização de técnicas estatísticas descritivas e modelos probabilísticos são fundamentais na realização de estudos voltados à análise do comportamento de vazões de determinada bacia. $\mathrm{O}$ presente estudo tem como objetivo analisar o comportamento da vazão, mínimas e máximas, de algumas seções do rio Paraíba do Sul. Para tanto, a metodologia empregada para a elaboração dos resultados compreende a lei de distribuição de frequências de Gumbel, cálculo do período de retorno e determinação do coeficiente de variação. Os dados foram trabalhados e analisados em planilhas do Excel, onde se constatou os períodos de retorno para os dados máximos e mínimos de vazão para a série histórica de três estações da bacia em questão. A partir da realização do estudo, destaca-se a importância da análise estatística na compreensão do comportamento hidrológico, como também, na gestão dos recursos.
\end{abstract}

Palavras Chave: vazões, período de retorno, Rio Paraíba do Sul.

\section{1 - INTRODUÇÃO}

O gerenciamento dos recursos hídricos atualmente é prioridade para a maioria dos governos e agências internacionais, a disponibilidade e a distribuição da água permeiam diversos conflitos e questões relativas à sua condição de escassez em quantidade ou qualidade. Desta maneira, em tempos em que a água deixou de ter valor natural e passou a ter valor econômico e político, o conhecimento hidrológico passou a ter fundamental importância para a gestão e controle dos recursos. Neste sentido, na atualidade tem se intensificado os estudos visando o entendimento do comportamento hidrológico, pressuposto básico para a gestão deste recurso.

Em estudos e pesquisas sobre recursos hídricos, as técnicas estatísticas são fundamentais para o processo de análise do comportamento de vazões. Para Valverde (2004), o estudo de distribuição de frequência de vazões máximas e mínimas necessita do uso de distribuições estatísticas. Segundo Leme (2003), os processos hidrológicos, como a vazão de um riosão aleatórios, sendo assim, o tratamento dos dados e informações com base estatística devem ser realizados a partir de distribuições teóricas de probabilidade aplicadas as séries hidrológicas. 
XVII Simpósio Brasileiro

de Geografia Fisica Aplicada

I Congresso Nacional

de Geografia Física

\section{OS DESAFIOS DA GEOGRAFIA FÍSICA NA FRONTEIRA DO CONHECIMENTO \\ Instituto de Geociências - Unicamp \\ Campinas - SP \\ 28 de Junho à 02 de Julho de 2017}

De acordo com Katz et al. (2002) e Queiroz \&Chaudhry (2006) vazões máximas e mínimas devem ser adequadamente tratadas do ponto de vista probabilístico. Sobre esta temática, Queiroz e Oliveira (2012) comentam:

A maioria dos modelos probabilísticos existentes em hidrologia utiliza a distribuição de Gumbel, onde se analisa os eventos extremos (vazões máximas e mínimas). Os resultados destas análises permitem entender o comportamento hidrológico da drenagem em análise, principalmente a predição dos eventos de cheias e de escassez hídrica. Estes dados são de suma importância no dimensionamento de obras hidráulicas (barragens, pontes, galerias, dentre outras) e ainda no planejamento da distribuição da água em períodos de escassez.

Vale ressaltar também, que a utilização das informações hidrológicas a partir da estimativa de probabilidades possuí caráter preventivo, com base em funções de densidade de probabilidade aplicadas aos valores observados as vazões possíveis de ocorrer. (SILVINO et al, 2007). Ainda, segundo Silvino (2007), as informações hidrológicas ligadas aos períodos de retorno de vazões máximas e mínimas são de fundamental importância para o dimensionamento de canais; dimensionamento de proteções contra cheias, pontes, vertedores, abastecimento de água de cidades, irrigação, projetos agrícolas, navegação, geração de energia, dentre outras atividades.

Diante do exposto,o objetivo deste trabalho é analisar o comportamento da vazão, mínimas e máximas, de algumas seções do rio Paraíba do Sul. Para tanto, serão aplicadas técnicas estatísticas descritivas e modelos probabilísticos para os dados de vazões máximas e mínimas das estaçõesSanta Branca (58099000), Pindamonhangaba (58183000) e Paraíba do Sul, (58380001)pertencentes à Bacia Hidrográfica do Rio Paraíba do Sul.

\section{Área de Estudos}

A área foco para o estudo compreende o Rio Paraíba do Sul com $1.130 \mathrm{~km}$ de extensão e percorre os estados de São Paulo, Minas Gerais e Rio de Janeiro. Possuí uma vazão em sua foz de aproximadamente $1.130 \mathrm{~m}^{3} / \mathrm{s}$ e seus principais afluentes são os Rios Jaguari, Paraibuna, Pomba e Muriaé pela margem esquerda e os Rios Piraí, Piabanha e Dois Rios pela margem direita. (ANA, 2017). Do ponto vista hidrológico, constitui-se em importante manancial de abastecimento público de água, suporte para atividades agropecuárias e geração de energia.

Segundo a classificação de Koppen, adotada universalmente e adaptada ao Brasil, o clima regional presente ao longo do Rio Paraíba do Sul varia entre Cwa, temperado úmido com invernos secos e verões quentes, Am, clima de monção e por fim, como Af, tropical úmido ou clima equatorial. 


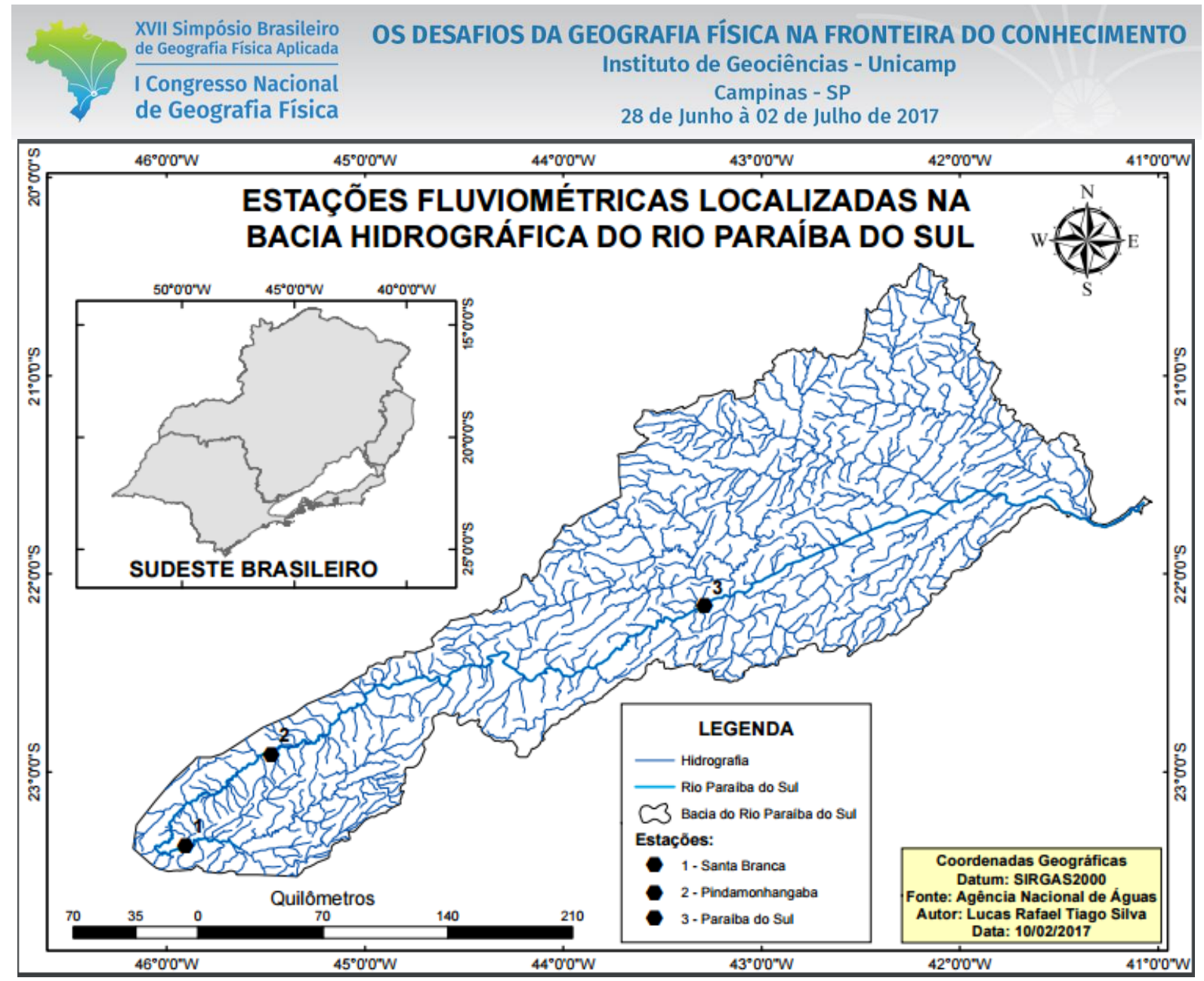

Figura 1 - Mapa de localização das estações fluviométricas utilizadas da Bacia Hidrográfica do Rio Paraíba do Sul.

Fonte: ANA (2017)

Organização: SILVA, 2017

\section{2 - METODOLOGIA}

Para o desenvolvimento do trabalho, foram utilizados dados de vazões máximas e mínimasdas estações fluviométricas de Santa Branca, código(58099000) e série histórica de 1952 a 2006, Pindamonhangaba, código (58183000) e série histórica de 1939 a 2007 e Paraíba do Sul, código(58380001) e série histórica de 1972 a 2006.Vale ressaltar que, para a realização dos cálculos probabilísticos e para a determinação dos períodos de retorno, foram utilizados os dados mais críticos de todas as séries, para tanto, na estação de Santa Branca a vazão máxima utilizada foi de $595 \mathrm{~m}$ 3/s e a vazão mínima de $17 \mathrm{~m}^{3} / \mathrm{s}$, na estação de Pindamonhangaba a vazão máxima foi de $688 \mathrm{~m}^{3} / \mathrm{s}$ e a vazão mínima de $50 \mathrm{~m} 3 / \mathrm{s}$ e por fim, na estação de Paraíba do sul a vazão máxima foi de $1450 \mathrm{~m} 3 / \mathrm{s}$ e a vazão mínima foi de $28 \mathrm{~m}^{3} / \mathrm{s}$.

Na tabulação, apresentação gráfica e análise estatística dos dados, foram utilizados os softwares Sistema Computacional para Análises Hidrológicas (Siscah 1.0) e Microsoft Office Excel. Nesta etapa metodológica, os dados foram coletados no banco de dados do Sistema de Informações Hidrológicas (Hidroweb) da Agência Nacional das Águas (ANA), posteriormente, os dados de vazões máximas e 


\section{OS DESAFIOS DA GEOGRAFIA FÍSICA NA FRONTEIRA DO CONHECIMENTO \\ Instituto de Geociências - Unicamp \\ Campinas - SP \\ 28 de Junho à 02 de Julho de 2017}

mínimas foram sistematizados no Siscah 1.0 e transferidos para o Microsoft Office Excel, onde foram realizadas todas as funções estatísticas para as três estações.

Os resultados correspondem àanálises paravalores críticos de vazão, para tanto, foi utilizada a lei de distribuição de frequências de Gumbel. Pela lei de Gumbel, a probabilidade de que se encontre um valor inferior a um determinado evento " $\mathrm{X}$ " pode ser calculado pela expressão:

$$
F(x)=e^{-e^{-b}}
$$

Onde: $\mathrm{e}=$ base dos logaritmos neperianos.

Para se calcular b, utiliza-se a expressão:

$\mathrm{b}=\alpha(\mathrm{x}-\mathrm{u})(2)$

Onde:

$\mathrm{x}=$ valor do evento a ser superado.

Para se calcular $\alpha$, utiliza-se a expressão:

$\alpha=\sigma \mathrm{y} / \mathrm{sx}(3)$

Onde:

$\sigma y=$ é a média de uma série de valores yi ( $\mathrm{i}=1$ a $\mathrm{N} ; \mathrm{N}=\mathrm{nr}$ de dados da amostra) que depende somente do número de dados. Valores encontram-se na tabela I.

$\mathrm{Sx}=$ desvio padrão da amostra

Para se calcular $\mu$, utiliza-se a expressão:

$\boldsymbol{u}=\bar{x}-\mu_{y} / \alpha(4)$

Onde:

$\overline{\boldsymbol{x}}=$ média aritmética da amostra

$\mu y=$ é o desvio padrão de uma série de valores yi ( $\mathrm{i}=1$ a $\mathrm{N} ; \mathrm{N}=\mathrm{nr}$ de dados da amostra) que depende somente do número de dados. Valores encontram-se na tabela I. 
XVII Simpósio Brasileiro de Geografia Fisica Aplicada

I Congresso Nacional de Geografia Física

\section{OS DESAFIOS DA GEOGRAFIA FÍSICA NA FRONTEIRA DO CONHECIMENTO \\ Instituto de Geociências - Unicamp \\ Campinas - SP \\ 28 de Junho à 02 de Julho de 2017}

Tabela I - Valores da média ( $\gamma$ n ) e do desvio-padrão (Sn ) da variável reduzida em função do tamanho da $\operatorname{amostra}(n)$

\begin{tabular}{|c|c|c|c|c|c|c|c|c|}
\hline $\mathrm{n}$ & $\gamma_{n}$ & $\$_{\mathrm{n}}$ & in & $\gamma_{m}$ & $S_{n}$ & $\mathrm{n}$ & $\gamma_{n}$ & $\mathrm{~S}_{\mathrm{w}}$ \\
\hline 10 & 0,4967 & 0,9573 & 45 & 0,5463 & 1,1519 & 73 & 0,5555 & 1,1881 \\
\hline 15 & 0,5128 & 1,0206 & 46 & 0,5468 & 1,1538 & 74 & 0,5557 & 1,1890 \\
\hline 20 & 0,5236 & 1,0628 & 47 & 0,5473 & 1,1557 & 75 & 0,5559 & 1,1898 \\
\hline 21 & $0, \$ 2 \$ 2$ & 1,0696 & 48 & 0,5477 & 1,1574 & 76 & 0,5561 & 1,1906 \\
\hline 22 & 0,5268 & 1,0754 & 49 & 0,5481 & 1,1590 & 7 & 0,5563 & 1,1915 \\
\hline 23 & 0,5283 & 1,0811 & 50 & 0,5485 & 1,1607 & 78 & 0,5565 & 1,1923 \\
\hline 24 & 0,5296 & 1,0864 & 51 & 0,5489 & 1,1623 & 79 & 0,5567 & 1,1930 \\
\hline 25 & 0,5309 & 1,0915 & 52 & 0,5493 & 1,1638 & 80 & 0,5569 & 1,1938 \\
\hline 26 & 0,5320 & 1,0961 & 53 & 0,5497 & 1,1658 & 81 & 0,5570 & 1,1945 \\
\hline 27 & 0,5332 & 1,1004 & 54 & 0,5501 & 1,1667 & 82 & 0,5572 & 1,1953 \\
\hline 28 & 0,5343 & 1,1047 & 55 & 0,5504 & 1,1681 & 83 & 0,5574 & 1,1960 \\
\hline 29 & 0,5353 & 1,1086 & 36 & 0,5508 & 1,1696 & 84 & 0,5576 & 1,1967 \\
\hline 30 & 0,5362 & 1,1124 & 57 & 0,5511 & 1,1708 & 85 & 0,5578 & 1,1973 \\
\hline 31 & 0,5371 & 1,1159 & 58 & 0,5515 & 1,1721 & 86 & 0,5580 & 1,1980 \\
\hline 32 & 0,5380 & 1,1193 & $\$ 9$ & 0,5518 & 1,1734 & 87 & 0,5581 & 1,1987 \\
\hline 33 & 0,5388 & 1,1226 & 60 & 0,5521 & 1,1747 & 88 & 0,5583 & 1,1994 \\
\hline 34 & 0,5396 & 1,1255 & 61 & 0,5524 & 1,1759 & 89 & 0,5585 & 1,2001 \\
\hline 35 & 0,5403 & 1,1285 & 62 & 0,5527 & 1,1770 & 90 & 0,5586 & 1,2007 \\
\hline 36 & 0,5410 & 1,1313 & 63 & 0,5530 & 1,1782 & 91 & 0,5587 & 1,2013 \\
\hline 37 & 0,5418 & 1,1339 & 64 & 0,5533 & 1,1793 & 92 & 0,5589 & 1,2020 \\
\hline 38 & 0,5424 & 1,1363 & 65 & 0,5535 & 1,1803 & 93 & 0,5591 & 1,2026 \\
\hline 39 & 0,5430 & 1,1388 & 66 & 0,5538 & 1,1814 & 94 & 0,5592 & 1,2032 \\
\hline 40 & 0,5436 & 1,1413 & 67 & 0,5540 & 1,1824 & 95 & 0,5593 & 1,2038 \\
\hline 41 & 0,5442 & 1,1436 & 68 & 0,5543 & 1,1834 & 96 & 0,5595 & 1,2044 \\
\hline 42 & 0,5448 & 1,1458 & 69 & 0,5545 & 1,1844 & 97 & 0,5596 & 1,2049 \\
\hline 43 & 0,5453 & 1,1480 & 70 & 0,5548 & 1,1854 & 98 & 0,5598 & 1,2055 \\
\hline \multirow[t]{2}{*}{44} & 0,5458 & 1,1499 & 71 & 0,5550 & 1,1863 & 99 & 0,5599 & 1,2060 \\
\hline & & & 72 & 0,5552 & 1,1873 & 100 & 0,5600 & 1,2065 \\
\hline
\end{tabular}

Fonte: Carvalho; Silva, 2006

Destaca-se outro importante modelo probabilístico no conhecimento hidrológico também utilizado para a análise do comportamento das vazões do presente estudo. O cálculo do período de retorno, muito utilizado em dimensionamento da capacidade de suporte de obras culturais. Para Pinto et al. (1976), Cruciani (1986) e Tucci (2000), o período de retorno representa o número médio de anos, durante o qual se espera que os valores sejam igualados ou superados ao menos uma vez.

Sendo assim, para se calcular que a frequência, ou período de retorno se apresentará umadeterminada vazão a cada nanosdevemos calcular bna expressão (1), resultando em:

$\mathrm{b}=-\ln (-\ln (\mathrm{F}(\mathrm{x})))(5)$

Onde:

$\mathrm{Ln}=$ logaritmo natural E finalmente, calcular $\mathrm{x}$ na equação (2):

$\mathrm{x}=\mathrm{b} / \alpha+\mathrm{u}(6)$ 


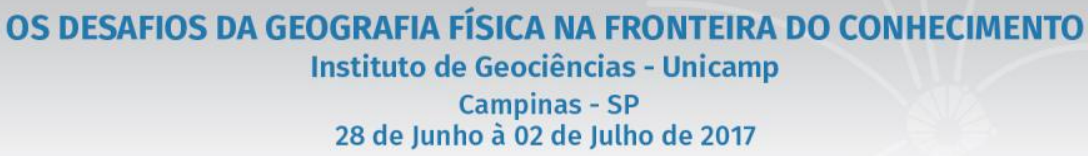

Outra metodologia empregada para o desenvolvimento do trabalho foi à determinação do coeficiente de variação $(\mathrm{CV})$, utilizado para analisar o comportamento dos dados e expressar a precisão do processo de análise. Quanto menor o coeficiente de variação mais homogêneo é o conjunto de dados. O CV pode ser considerado baixo quando for menor ou igual a $25 \%$, porém esse valor pode variar de acordo com a aplicação dos dados.

Sendo assim, o coeficiente de variação pode ser calculado pela seguinte expressão:

$\mathrm{CV}=s / \bar{x}$

Onde:

$\mathrm{s}=$ desvio padrão

$\mathrm{x}=$ média aritmética da amostra

\section{3 - RESULTADOS}

A partir da análise estatística dos dados foram constatados valores referentes ao período de retorno para vazões críticas das três estações em análise e valores para o coeficiente de variação de cada uma das três séries de dados.

Os valores estatísticos de probabilidade, períodos de retorno das vazões máximas e mínimas das respectivas estações estão sumariados na tabela II.

Tabela II - Vazões críticas e períodos de retorno.

\begin{tabular}{|l|c|c|c|c|}
\hline Estações e Série Histórica & Vazão Máxima (m³ $/ \mathrm{s})$ & Período de retorno (anos) & Vazão Mínima (m³ $/ \mathrm{s})$ & Período de retorno (anos) \\
\hline Santa Branca: 1952-2006 & 595 & 125 & 17 & 174 \\
\hline Pindamonhangaba: $1939-2007$ & 688 & 25 & 50 & 98 \\
\hline Paraiba so Sul: $1972-2006$ & 1450 & 20 & 28 & 21 \\
\hline
\end{tabular}

Org. dos autores

A partir dos dados da tabela II, é possível compreender a variação do comportamento das vazões ao longo das séries históricas em destaque. Para a Estação de Santa Branca o período de retorno foi de 125 anos para a vazão máxima de $595 \mathrm{~m}^{3} / \mathrm{s}$ e de 174 anos para a vazão mínima de $17 \mathrm{~m}^{3} / \mathrm{s}$. Para a estação de Pindamonhangaba ressalta-se o período de retorno de 25 anos para a vazão máxima de $688 \mathrm{~m}^{3} / \mathrm{s}$ e assumindo maior destaque, para a estação de Paraíba do Sul determinou-se um período de retorno de 20 anos para uma vazão máxima de $1450 \mathrm{~m} 3 / \mathrm{s}$ e de 21 anos para uma vazão mínima de 28 $\mathrm{m}^{3} / \mathrm{s}$. 
XVII Simpósio Brasileiro

de Geografia Fisica Aplicada

I Congresso Nacional

de Geografia Física

\section{OS DESAFIOS DA GEOGRAFIA FÍSICA NA FRONTEIRA DO CONHECIMENTO \\ Instituto de Geociências - Unicamp \\ Campinas - SP \\ 28 de Junho à 02 de Julho de 2017}

Para um melhor entendimento sobre o comportamento de vazões entre as três estações foram calculados também, os coeficientes de vazão (mínimas e máximas), cujos valores encontram-se nas tabelas III e IV, respectivamente.

Tabela III - Variação para vazões mínimas.

\begin{tabular}{|l|c|c|c|}
\hline Estações e Série Histórica & Média Aritmética & Desvio Padrão & Coeficiente de Variação (\%) \\
\hline Santa Branca: $1952-2006$ & 34.8 & 9.7 & $28 \%$ \\
Pindamonhangaba: $1939-2007$ & 78.4 & 15.9 & $20 \%$ \\
\hline Paraiba do Sul: $1972-2006$ & 60.5 & 22.4 & $37 \%$ \\
\hline
\end{tabular}

Org. dos autores

Tabela IV - Variação para vazões máximas.

\begin{tabular}{|l|c|c|c|}
\hline Estações e Série Histórica & Média Aritmética & Desvio Padrão & Coeficiente de Variação (\%) \\
Santa Branca: 1952-2006 & 199.8 & 107.6 & $54 \%$ \\
Pindamonhangaba: 1939-2007 & 397.7 & 129.3 & $30 \%$ \\
\hline Paraiba do Sul: 1972-2006 & 809.8 & 291.2 & $36 \%$ \\
\hline
\end{tabular}

Org. dos autores

A partir do coeficiente de variação podemos analisar quais séries de dados entre as estações estão mais homogêneos e estáveis em relação à média. Para os dados de vazões mínimas, representados pela tabela 3, a série de dados mais homogênea e estável compreende a estação de Pindamonhangaba, CV de $20 \%$ e série histórica (1939-2007). Para as outras estações, observa-se uma menor consistência dos dados, CV de $28 \%$ e 37\%, para Santa Branca e Paraíba do Sul, respectivamente.

Na variação do coeficiente para as vazões máximas, a série de dados mais homogênea também foi da estação de Pindamonhangaba, com CV de $30 \%$. Para a estação de Santa Branca a variação foi de $50 \%$ e na estação de Paraíba do Sul de $36 \%$.

Portanto, a partir da análise dos resultados destaca-se a importância das funções estatísticas na compreensão do comportamento hidrológico de determinada drenagem. A partir deste estudo, a utilização das técnicas estatísticas descritivas e das funções de probabilidade, foi possível constatar o período de retorno de vazões críticas para três estações da Bacia Hidrográfica do Rio Paraíba do sul. A determinação destes valores podem ser úteis para diversas atividades ou ações humanas presentes na área, sendo assim, vale ressaltar que, a correlação dos dados e dos resultados com o comportamento físico e social de determinada área é fundamental para gestão correta dos recursos. 
XVII Simpósio Brasileiro

de Geografia Fisica Aplicada

I Congresso Nacional

de Geografia Física

OS DESAFIOS DA GEOGRAFIA FÍSICA NA FRONTEIRA DO CONHECIMENTO

Instituto de Geociências - Unicamp

Campinas - SP

28 de Junho à 02 de Julho de 2017

\section{4 - CONSIDERAÇÕES FINAIS}

A utilização das técnicas estatísticas é fundamental na realização de estudos voltados à análise do comportamento de vazões de determinada bacia. Além do potencial econômico, o entendimento do comportamento hidrológico é pressuposto básico para a gestão dos recursos hídricos.

A partir da realização do estudo, conclui-se que os períodos de retorno determinados podem ser utilizados como mecanismos e ferramentas para as atividades humanas da área e a metodologia empregada teve um papel didático imensurável, no que se refere à análise estatística e compreensão dos eventos.

Enfim, os resultados obtidos foram satisfatórios, no entanto, ressalta-se que foi possível encontrar algumas falhas nos dados coletados durante a análise.

\section{5 - REFERÊNCIAS}

QUEIROZ, A. T. E OLIVEIRA, L. A. "Relação entre produção e demanda hídrica na bacia do rio Uberabinha, estado de Minas Gerais, Brasil." Revista Sociedade \& Natureza 25.1 (2013).

VALVERDE, A. E. LACERDA; LEITE, H. GARCIA; SILVA, D. D. DA; PRUSKI, F. FALCO.

Momentos-l: teoria e aplicação em hidrologia. R. Árvore, Viçosa-MG, v.28, n.6, p.927-933, 2004.

LEME, E.J.A. Hidrologia estatística da vazão mínima do rio Jaguari Mirim. Ecossistema, Espírito Santo do Pinhal, v. 27, n. 1,2, p. 77-82, 2002.

KATZ, R.W.; PARLANGE, M.B.; NAVEAU, P. Statisticsof extremes in hydrology. Advances in WaterResources, Amsterdam, v.25, p.1287-1304, 2002.

QUEIROZ, M.M.F. de; CHAUDHRY, F.H. Análise de eventos hidrológicos extremos usando a distribuição GEV e momentos LH. Revista Brasileira de Engenharia Agrícola e Ambiental, Campina Grande, v.10, n.2, p.381- 389, 2006.

SILVINO, A. N. O.; SILVEIRA, A.; MUSIS, C. R.; WYREPKOWSKI, C. C. Determinação de vazões extremas para diversos períodos de retorno para o Rio Paraguai utilizando métodos estatísticos. Geociências, São Paulo, v. 26, n.4, p. 369-378, 2007.

CARVALHO, D. F.; SILVA, L. D. B. Hidrologia. Rio de Janeiro: Universidade Federal Rural do Rio de Janeiro, 2006. 115 p. Disponível em , acesso em 10 de nov. de 2011.

AGÊNCIA NACIONAL DE ÁGUAS (ANA). HIDROWEB - Sistema de Informações Hidrológicas. Disponível em <http://hidroweb.ana.gov.br/>, acesso em 10 de janeiro de 2017. 